\title{
ON THE STRONGLY EXTREME POINTS OF CONVEX BODIES IN SEPARABLE BANACH SPACES
}

\author{
B. V. GODUN, BOR-LUH LIN, AND S. L. TROYANSKI
}

(Communicated by William J. Davis)

\begin{abstract}
Every separable Banach space admits an equivalent norm such that the unit ball with respect to this norm has at most countably many strongly extreme points. Every separable nonreflexive Banach space can be renormed so that its unit ball has at most countably many weakly strongly extreme points.
\end{abstract}

Let $X$ be an infinite-dimensional Banach space. A well-known result of Linderstrass and Phelps [LP] shows that if $X$ is reflexive, then every bounded closed convex body in $X$ has uncountably many extreme points. On the other hand, according to a theorem of Fonf [F], if the unit ball of $X^{*}$ contains only countably many extreme points, then $c_{0}$ is isomorphic to a subspace of $X$. This shows that the cardinality of the set of extreme points of bounded closed convex bodies of a Banach spaces is related to its linear topological properties. In this paper we show that the cardinality of the set of strongly extreme points of bounded closed convex bodies of a separable Banach space is not related to its linear topological property by showing the every separable Banach space admits an equivalent norm such that the unit ball under the new norm has at most countably many strongly extreme points. Furthermore, using the similar argument, it is proved that every separable nonreflexive Banach space admits an equivalent norm so that the unit ball admits at most countably many weakly strongly extreme points. As a consequence, it follows that a separable Banach space is reflexive if and only if every bounded closed convex body in the space has uncountably many weakly strongly extreme points.

Let $C$ be a bounded closed convex set of a Banach space $X$. A point $x$ in $C$ is called a (weakly) strongly extreme point [KR] of $C$ if for any sequences $\left\{y_{n}\right\},\left\{z_{n}\right\}$ in $C, \lim _{n}\left\|x-\left(y_{n}+z_{n}\right) / 2\right\|=0$ implies $\lim _{n}\left\|y_{n}-z_{n}\right\|=0$. (weak $-\lim _{n}\left(y_{n}-z_{n}\right)=0$; that is, $\left(y_{n}-z_{n}\right)$ converges weakly to 0 .) $x$ is called a weak*-extreme point of $C$ if $x$ is an extreme point of the weak*closure of $C$ in $X^{* *}$. It is clear that every strongly extreme point of $C$ is a

Received by the editors May 28, 1990 and, in revised form, September 4, 1990.

1980 Mathematics Subject Classification (1985 Revision). Primary 46B20.

Key words and phrases. Strongly extreme points, weakly strongly extreme points, weak*-extreme points, reflexive Banach spaces, equivalent norm.

The third author's work was supported by the Science Committee of Bulgaria, \#54/2503, 1987.

This paper was presented in a special session on Banach Spaces-Functional Analysis, American Mathematical Society meeting at Denton, Texas November 2, 1990. 
weakly strongly extreme point. In $[K R]$ it is proved that every strongly extreme point of $C$ is a weak*-extreme point of $C$. In fact, in a manner similar to the proof in [KR], it follows that every weakly strongly extreme point of $C$ is a weak*-extreme point of $C$. It is easy to see that the converse is also true (e.g. using [R1, Proposition 1.2]); i.e. weak*-extreme point and weakly strongly extreme point are identical.

Let $E$ be a subspace of $X^{*}$. The number

$$
r(E)=\inf _{\substack{x \in X \\\|x\|=1}} \sup _{\substack{x^{*} \in E \\\left\|x^{*}\right\|=1}}\left|x^{*}(x)\right|
$$

is called the Dixmier characteristic of $E$.

Lemma. Let $X$ be a separable nonreflexive Banach space. Then there exists a separable subspace $E$ in $X^{*}$ with $r(E)>0$ and a sequence $\left\{x_{n}\right\}$ in $X$ such that $\left\|x_{n}\right\|=1, n \in \mathbb{N}, \lim _{n} x^{*}\left(x_{n}\right)=0$ for all $x^{*}$ in $E$ but 0 is not in the weak closure of $\left\{x_{n}\right\}$.

Proof. Let $x^{* *} \in X^{* *} \backslash X,\left\|x^{* *}\right\|=1$ and $\left[x^{* *}\right]_{\perp}=\left\{x^{*}: x^{*} \in X^{*}, x^{* *}\left(x^{*}\right)=\right.$ $0\}$. It is clear that $r\left(\left[x^{* *}\right]_{\perp}\right) \leq \inf _{x \in X,\|x\|=1} d\left(x,\left[x^{* *}\right]\right)$ where $\left[x^{* *}\right]$ is the subspace spanned by $x^{* *}$, and using Helly theorem, for example, it follows that $r\left(\left[x^{* *}\right]_{\perp}\right)=\inf _{\|x\|=1, x \in X} d\left(x,\left[x^{* *}\right]\right)>0$. Since $X$ is separable, there exists a separable subspace $E$ in $\left[x^{* *}\right]_{\perp}$ such that $r(E)=r\left(\left[x^{* *}\right]_{\perp}\right)>0$. Let $\left\{x_{n}^{*}\right\}$ be a dense sequence in $E$ and let $x^{*}$ in $X^{*}$ with $x^{* *}\left(x^{*}\right)>1$. Since the unit ball of $X$ is weak* dense in the unit ball of $X^{* *}$, for each $n \in \mathbb{N}$, there exists $x_{n}$ in $X,\left\|x_{n}\right\|=1,\left|x_{k}^{*}\left(x_{n}\right)\right|<\frac{1}{n}, k=1,2, \ldots, n$, and $x^{*}\left(x_{n}\right)>1$. Then $E$ and $\left\{x_{n}\right\}$ satisfy the conditions in the lemma.

Theorem 1. Let $X$ be a separable Banach space. Then there is an equivalent norm $|\cdot|$ on $X$ such that the unit ball of $(X,|\cdot|)$ has at most countably many strongly extreme points.

Proof. As in the last part of the proof of the lemma, there is a subspace $E$ in $X^{*}$ with countable dimension, $r(E)=1$, and a sequence $\left\{x_{n}\right\},\left\|x_{n}\right\|=1$, in $X$ such that $\sigma(X, E)-\lim _{n} x_{n}=0$ where $\sigma(X, E)$ is the weak topology on $X$ induced by $E$. Let $\left\{y_{n}\right\}$ be a dense sequence in the unit ball $B_{X}$ of $X$. Put $z_{n}=x_{n}+y_{n}, w_{n}=x_{n}-y_{n}, n \in \mathbb{N}$, and let $T=\left\{ \pm z_{n}, \pm w_{n}\right\}_{n \in \mathbb{N}}$ and $U=\operatorname{clco}(T)$. Let $|\cdot|$ be the Minkowski functional of $U$. Then it is easy to see that $|\cdot|$ is an equivalent norm on $X$ and

$$
\left|x^{*}\right|=\sup _{t \in T}\left|x^{*}(t)\right|, \quad x^{*} \in X^{*} .
$$

To show that $U$ has at most countably many strongly extreme points it suffices to show that all the strongly extreme points of $U$ are in $T$. Since every strongly extreme point of $U$ is a weak*-extreme point of $U$, it remains to show that if $x$ is a weak*-extreme point of $U$ and $x \notin T$, then there is a subsequence $\left\{x_{n_{k}}\right\}$ of $\left\{x_{n}\right\}$ such that $\lim _{k}\left|x \pm x_{n_{k}}\right|=1$ for some choice of signs \pm 1 .

Let $x$ be a weak*-extreme point of $U$. Then the family of slices $S\left(x^{*}, \varepsilon\right)=$ $\left\{y: y \in U, x^{*}(y)>1-\varepsilon\right\},\left|x^{*}\right|=1, \varepsilon>0$ forms a neighborhood base for $x$ in the weak topology on $U$ (see e.g. [R2, Lemma 1.3]). According to (1), $S\left(x^{*}, \varepsilon\right) \cap T \neq \phi$ for all $\left|x^{*}\right|=1$ and $\varepsilon>0$. Therefore $x \in$ weak $-\operatorname{cl}(T) \subset$ $\sigma(X, E)-\operatorname{cl}(T)$. Since $x \notin T$ and $\sigma(X, E)$ is metrizable, without loss of generality we may assume that there is a sequence $\left\{m_{k}\right\}$ such that $\sigma(X, E)-$ 
$\lim z_{m_{k}}=x$. Since $\sigma(X, E)-\lim _{k} x_{m_{k}}=0$, we get $\sigma(X, E)=\lim _{k} y_{m_{k}}=x$. Since $r(E)=1, \lim _{k}\left\|y_{m_{k}}\right\| \geq\|x\|$ and so $\|x\| \leq 1$. Now $\left\{y_{n}\right\}$ is norm dense in $B_{X}$, and it follows that there is a sequence $\left\{y_{n_{k}}\right\}$ such that $\lim _{k}\left\|y_{n_{k}}-x\right\|=0$ and so $\lim _{k}\left|y_{n_{k}}-x\right|=0$. Therefore $\lim _{k}\left|x \pm x_{n_{k}}\right|=\lim _{k}\left|y_{n_{k}} \pm x_{n_{k}}\right|=1$.

Theorem 2. Let $X$ be a separable nonreflexive Banach space. Then $X$ admits an equivalent norm such that the unit ball under the new norm has at most countably many weakly strongly extreme points, i.e. weak*-extreme points.

Proof. By Lemma and renorming, there exists a separable subspace $E$ in $X^{*}$ with $r(E)=1$ and a sequence $\left\{x_{n}\right\},\left\|x_{n}\right\|=1$, in $X$ such that $\sigma(X, E)-$ $\lim _{n} x_{n}=0$ but $0 \notin$ weak $-\operatorname{cl}\left\{x_{n}\right\}$. As in the proof of Theorem 1, let $U$ be the unit ball under the equivalent norm $|\cdot|$. Then $U$ is a bounded closed convex body in $X$. We claim that $T$ contains all weakly strongly extreme points of $U$, and so $U$ has at most countably weakly strongly extreme points.

Suppose $x$ is a weakly strongly extreme point of $U$ that is not in $T$. By [KR] $x$ is a weak*-extreme point of $U$. As proved in Theorem 1 , it follows that there is a subsequence $\left\{x_{n_{k}}\right\}$ of $\left\{x_{n}\right\}$ such that $\lim _{k}\left|x \pm x_{n_{k}}\right|=1$. Since $x$ is a weakly strongly extreme point of $U$, weak $-\lim _{k} x_{n_{k}}=0$ and so $0 \in$ weak $-\operatorname{cl}\left\{x_{n}\right\}$, which is a contradiction.

Corollary. Let $X$ be a separable Banach space. Then $X$ is reflexive if and only if every bounded closed convex body in $X$ has uncountably many weakly strongly extreme points, i.e. weak*-extreme points.

Proof. Suppose $X$ is separable and nonreflexive. By Theorem 2, the unit ball of some equivalent norm in $X$ is a bounded closed convex body in $X$ with at most countably many weakly strongly extreme points.

Conversely, if $X$ is reflexive, by the theorem of Linderstrass and Phelps [LP], every bounded closed convex body $C$ has uncountably many extreme points. Since $C$ is weakly compact, $x$ is an extreme point of $C$ if and only if $x$ is a weak*-extreme point of $C$.

Finally, let us remark that the problem raised in [LP] regarding whether every separable Banach space contains a bounded closed convex body with countably many exposed points is still unsolved.

\section{REFERENCES}

[F] V. Fonf, One property of Linderstrass-Phelps spaces, Funct. Anal. Appl. 13 (1979), 66-67.

[KR] K. Kunen and H. P. Rosenthal, Martingale proofs of some geometrical results in Banach space theory, Pacific J. Math. 100 (1982), 153-175.

[LP] J. Lindenstrauss and R. R. Phelps, Extreme points properties of convex bodies in reflexive Banach spaces, Israel J. Math. 6 (1968), 39-48.

[R1] H. P. Rosenthal, On non-norm-attaining functionals and the equivalence of the weak ${ }^{*}$-KMP with the RNP, Longhorn Notes, The University of Texas at Austin, 1985/86, pp. 1-12. [R2] $\frac{}{1-58 .}$. On the structure of non-dentable closed bounded convex sets, Adv. in Math. 70 (1988),

Kharkov Institute for Communal Constructions, Engineers, 12, Revoluci Str., 31002 , KHARKov, Union OF SOVIET Socialist RePUblics

Department of Mathematics, The University of Iowa, Iowa City, Iowa 52242

Department of Mathematics, Sofia University, 5 A. Ivanov Blvd., BG 1126, Sofia, BULGARIA 